\title{
Hitting Ras where it counts: Ras antagonism in the basolateral amygdala inhibits long-term fear memory
}

\author{
Stephen M. Merino ${ }^{1}$ and Stephen Maren ${ }^{1,2}$ \\ ${ }^{1}$ Neuroscience Graduate Program and \\ ${ }^{2}$ Department of Psychology, University of Michigan, 525 E. University Ave., Ann Arbor, MI 48109-1109, USA
}

Keywords: farnesylthiosalicylic acid, freezing, MAPK, rat, synaptic plasticity

\begin{abstract}
Several studies have implicated the Ras/mitogen-activated protein kinase (MAPK) pathway in Pavlovian fear conditioning. RasGRF1 knockout mice show significant deficits in acquisition of long-term fear memories and long-term potentaition (LTP) in the basolateral amygdala (BLA). MAPK kinase inhibition also impairs fear conditioning and amygdaloid LTP. However, there is no direct evidence to date for the involvement of Ras itself in fear conditioning. To address this issue, we examined the effects of intra-amygdala infusions of the selective Ras antagonist farnesylthiosalicylic acid (FTS) on the acquisition and expression of conditional freezing in rats. Microinfusions of FTS into the BLA prior to contextual fear conditioning significantly impaired acquisition of long-term contextual fear memory in a dose-dependent manner. Post-training FTS infusions had no effect on acquisition of long-term fear memory. The effects of FTS on fear conditioning were specific for the BLA. Finally, intra-amygdala infusions of FTS inhibited MAPK activation in BLA. Collectively, these results provide further evidence for the involvement of amygdaloid Ras in the acquisition of long-term conditional fear memory.
\end{abstract}

\section{Introduction}

Substantial evidence indicates that the basolateral amygdala (BLA) is a critical locus for the plastic changes underlying Pavlovian fear conditioning (Fanselow \& Ledoux, 1999; Maren, 2001; Schafe et al., 2001; Maren \& Quirk, 2004). A likely cellular mechanism for Pavlovian fear conditioning is long-term potentiation (LTP) in the BLA (Maren, 1999; Blair et al., 2001). LTP in the BLA can be induced by stimulation of afferents that carry either contextual (Maren \& Fanselow, 1995) or auditory information (Clugnet \& Ledoux, 1990; Rogan \& Ledoux, 1995; Huang \& Kandel, 1998). Fear conditioning causes LTP-like changes in the BLA of awake, behaving rats (Rogan et al., 1997) and in amygdala slices from fear-conditioned rats (McKernan \& Shinnick-Gallagher, 1997). Furthermore, infusions of NMDA receptor antagonists into the BLA impair both fear conditioning (Miserendino et al., 1990; Lee \& Kim, 1998; Maren et al., 1996) and LTP induction in the BLA (Maren \& Fanselow, 1995; Huang \& Kandel, 1998; Bauer et al., 2002).

Important advances have been made in our understanding of the molecular and cellular mechanisms underlying fear learning and memory. Increasing evidence suggests that the Ras/mitogen-activated protein kinase (MAPK) pathway is a vital component of amygdaloid plasticity and fear memory (Mazzuccheli \& Brambilla, 2000; Adams \& Sweatt, 2002). A role for the Ras/MAPK pathway in synaptic plasticity and memory was first demonstrated in the hippocampus (English \& Sweatt, 1996, 1997; Blum et al., 1999; Impey et al., 1999). Several recent studies point to a role for the Ras/MAPK pathway in amygdala-dependent learning and memory. Mice lacking RasGRF, a guanine-nucleotide exchange factor and activator of Ras, exhibit

Correspondence: Dr S. Maren, ${ }^{2}$ Department of Psychology, as above. E-mail: maren@umich.edu

Received 6 May 2005, revised 4 November 2005, accepted 7 November 2005 significant deficits in acquisition of long-term fear memory and impaired LTP in the BLA (Brambilla et al., 1997). Pharmacological inhibition of MAPK activation also causes deficits in fear conditioning and amygdaloid LTP (Schafe et al., 2000a). Recently, it was demonstrated that mice lacking the Ras effector Rin1, which functions through diversion of Ras signaling to alternate pathways, exhibit enhanced amygdaloid LTP and elevated amygdala-dependent aversive memory (Dhaka et al., 2003).

However, despite strong evidence implicating the Ras/MAPK pathway in fear conditioning, there is no direct evidence to date for the involvement of amygdaloid Ras in fear conditioning. Previous studies have implicated only upstream (RasGRF) and downstream (MAPK, Rin1) components of the pathway. To address this, we examined the effects of intra-amygdala infusion of the selective Ras antagonist farnesylthiosalicylic acid (FTS) on the acquisition and expression of conditional freezing in rats. FTS is an $S$-prenyl cysteine analog that dislodges Ras from the cell membrane, thus facilitating its degradation and reducing cellular levels of the protein (Haklai et al., 1998; Niv et al., 1999; Kloog \& Cox, 2004). Our results indicate that Ras function in the BLA is required for the acquisition but not expression of long-term conditional fear memory, lending further support to the notion that the Ras/MAPK pathway is required for consolidation of fear memory.

\section{Methods}

\section{Subjects}

Adult male Long-Evans rats (200-224 g) were obtained from a commercial supplier (Harlan Sprague-Dawley, Indianapolis, IN, USA) and individually housed in standard Plexiglas hanging cages on a $14: 10$-h light-dark cycle (lights on at 07:00 h). Food and water 
were provided ad libitum. Rats were handled for $30 \mathrm{~s}$ per day for 5 days to acclimate them to the experimenter. All experiments were carried out in accordance with guidelines approved by the University of Michigan University Committee on Use and Care of Animals.

\section{Surgery}

One week before behavioral testing, rats were implanted with guide cannulas (26 gauge; Plastics One, Roanoke, VA, USA) aimed at the BLA, central nucleus of the amygdala (CEA) or caudate-putamen (CPU). Rats were anesthetized with sodium pentobarbital $(65 \mathrm{mg} / \mathrm{kg}$ i.p.), and were administered atropine methyl nitrate $(0.4 \mathrm{mg} / \mathrm{kg}$ i.p. $)$ to prevent airway obstruction. After mounting in a stereotaxic apparatus (Kopf Instruments, Tujunga, CA, USA), the scalp was incised and retracted, and lambda and bregma were placed in the same horizontal plane. Small burr holes were drilled for bilateral placement of guide cannulas $1 \mathrm{~mm}$ above the BLA $(2.3 \mathrm{~mm}$ posterior to bregma, $5.0 \mathrm{~mm}$ lateral to the midline, $5.8 \mathrm{~mm}$ ventral to dura), CEA (2.0 $\mathrm{mm}$ posterior to bregma, $4.0 \mathrm{~mm}$ lateral to the midline, $5.7 \mathrm{~mm}$ dorsal to dura) or CPU $(2.3 \mathrm{~mm}$ posterior to bregma, $5.0 \mathrm{~mm}$ lateral to the midline, $3.7 \mathrm{~mm}$ ventral to dura), and placement of three small jeweler's screws. The guide cannulas were lowered, and dental acrylic was applied to the skull to hold the cannulas in place. After surgery, obturators (33 gauge; Plastics One) were inserted into the guide cannulas, and the rats were returned to their home cages. The obturators were replaced every other day during the week of recovery.

\section{Behavioral apparatus}

Eight identical observation chambers $(30 \times 24 \times 21 \mathrm{~cm}$; MED-Associates Inc., Burlington, VT, USA) were used for all training and testing. The chambers were constructed of aluminum (two side walls) and Plexiglas (rear wall, ceiling and hinged front door), and situated in sound-attenuating cabinets in an isolated room. The floor of each chamber consisted of 19 stainless steel rods (4 mm in diameter) spaced $1.5 \mathrm{~cm}$ apart (centre-to-centre). The rods were wired to a shock source and solid-state grid scrambler (MED-Associates Inc.) for the delivery of footshock unconditional stimuli (USs). A speaker for delivering acoustic stimuli was mounted to a grating on one wall of each chamber.

Each conditioning chamber rested on a load-cell platform that recorded chamber displacement in response to each rat's motor activity. The output of each chamber's load cell was amplified at a level that was previously determined to optimize the detection of freezing behavior. The load-cell amplifier output from each chamber was digitized at $5 \mathrm{~Hz}$ (yielding 300 observations per minute per rat) and acquired on-line using Threshold Activity Software (MEDAssociates Inc.). The average raw load-cell output was used to quantify activity. Freezing was quantified by calculating the number of observations below a freezing threshold. Freezing was scored only if a rat was immobile for $1 \mathrm{~s}$ or longer; thus freezing was scored only for five or more contiguous observations. For each session, the freezing observations were converted to a percentage of total observations. The chambers were cleaned with a $1 \%$ ammonium hydroxide solution, and stainless steel pans with a thin layer of the same solution were placed under the grid floors before rats were placed in the chambers. Illumination was provided by both the room lights and a stall stimulus light $(15 \mathrm{~W})$ in each chamber, and background noise (65 dB, A-scale) was supplied by ventilation fans in each chest. This collection of stimuli is referred to as Context A. Contextual fear conditioning occurred in Context A. For the auditory fear conditioning experiment
(Exp. 3), training and context testing occurred in Context $\mathrm{A}$ and tone fear testing occurred in Context B. For Context B, a 1\% acetic acid solution was used to clean the chambers and placed under the pans in the grid floor. In addition, illumination was provided only by a $15-\mathrm{W}$ red light in the room and the ventilation fans were turned off.

\section{Experiment 1a: dose-dependent effect of intra-BLA FTS on contextual fear conditioning}

The subjects were 32 adult male rats. All rats received bilateral cannula implants aimed at the BLA. After 1 week of recovery from surgery, the rats were habituated to the infusion procedure and conditioning context. The rats were transported to the laboratory and given a mock intracranial infusion, for which the obturators were removed and replaced, and an infusion pump (Harvard Apparatus, South Natick, MA, USA) was turned on for $1 \mathrm{~min}$ at a rate of $0.25 \mu \mathrm{L} / \mathrm{min}$ but no fluid was infused into the brain. The pumps were then turned off and the rats were returned to their home cages. Later that day, the rats were placed in the conditioning chambers (Context A) for 6 min but received no shocks. On the following day, the rats underwent contextual fear conditioning. The rats were transported to the laboratory and injection cannulas (Plastics One) extending $1 \mathrm{~mm}$ past the guide cannulas and attached to the pump with polyethylene tubing (PE-20; A-M Systems, Inc., Carlsborg, WA, USA) were inserted. Rats were administered an infusion of either vehicle $(100 \%$ DMSO; Sigma, St Louis, MO, USA) or FTS in varying concentrations (1.2, 12 or $120 \mu \mathrm{g} / \mu \mathrm{L}$; BIOMOL, Plymouth Meeting, PA, USA) for $30 \mathrm{~s}$ at a rate of $0.5 \mu \mathrm{L} / \min (0.25 \mu \mathrm{L}$ per side $)$. This resulted in doses of $0(n=8), 0.3(n=8), 3(n=8)$ or $30(n=8) \mu \mathrm{g}$ per side. Immediately after infusions the rats were returned to their home cages and $15 \mathrm{~min}$ later were brought to the laboratory and placed in the conditioning chambers. Three minutes after placement into the conditioning chambers (Context $\mathrm{A}$ ), the rats received five unsignaled footshocks ( $2 \mathrm{~s}, 1.0 \mathrm{~mA}, 1$-min intershock interval). Sixty seconds after the final shock the rats were returned to their home cages. Fear conditioning to the context was assessed by returning the rats to the conditioning chambers $2-4 \mathrm{~h}$ later and assessing freezing during an 8-min retention test.

\section{Experiment 1b: effect of intra-BLA FTS on expression of memory for contextual fear conditioning}

Twenty rats from Experiment 1a were given new group assignments, counterbalancing for previous group assignment. Twenty-four hours after the retention test the rats were re-trained drug-free, using the same parameters as in Experiment 1 . Three days after re-training the rats received an infusion of either $30 \mu \mathrm{g}$ FTS per side $(n=10)$ or vehicle $(n=10)$ into the BLA and were returned to their home cages for $15 \mathrm{~min}$ after which they were returned to the conditioning chamber for an 8-min retention test.

\section{Experiment 2: effect of pretraining FTS infusions into the BLA on acquisition of short-term fear memory}

The subjects were 14 adult male rats. All rats received bilateral guide cannula implants aimed at the BLA. As in Experiment 1, on the day before training, rats underwent mock infusions. On the following day, the rats received bilateral infusions of either $30 \mu \mathrm{g}$ FTS per side or vehicle at a rate of $0.25 \mu \mathrm{L} / \mathrm{min}$ for $1 \mathrm{~min}$. Injectors were left in for an additional minute before removal. Rats were then returned to their home cages for $15 \mathrm{~min}$, after which they were transported to the 
laboratory for auditory fear conditioning. Three minutes after being placed in the chambers, rats received ten tone $(30 \mathrm{~s}, 85 \mathrm{~dB}$ tone, $2 \mathrm{kHz})$ - shock (1 mA, $2 \mathrm{~s})$ pairings [1 min intertrial interval (ITI)]. The rats were returned to their home cages after training and were retrieved $1 \mathrm{~h}$ later for a short-term memory test, which consisted of a 2-min pretrial period then a 2-min continuous tone $(85 \mathrm{~dB}, 2 \mathrm{kHz})$. On the following day, a long-term memory test was administered, consisting of a 2 -min pretrial period and a 6 -min continuous tone (85 dB, $2 \mathrm{kHz})$.

\section{Experiment 3: effect of post-training FTS infusions into the BLA on acquisition of long-term fear memory}

The subjects were 33 adult male rats. All rats received bilateral guide cannula implants aimed at the BLA. As in Experiment 1, on the day before training, rats underwent mock infusions and pre-exposure to the conditioning chambers as described for Experiment 1. On the following day, the rats were transported to the laboratory for contextual fear conditioning. Three minutes after being placed in the chambers, rats received three unsignaled footshocks $(2 \mathrm{~s}, 1.0 \mathrm{~mA}$, $1 \mathrm{~min}$ ITI). After the fear conditioning session, the rats received bilateral infusions of either $30 \mu \mathrm{g}$ FTS per side or vehicle $(n=8$, split among each time point) at one of three post-training time points: immediately $(n=9), 1 \mathrm{~h}(n=8)$ or $6 \mathrm{~h}(n=8)$ after training. Rats receiving infusions 1 or $6 \mathrm{~h}$ after training were returned to their home cages after training and retrieved at the appropriate times for infusions. On the following day, fear conditioning to the context was assessed by returning the rats to the conditioning chambers and measuring freezing during an 8-min retention test.

\section{Experiment 4: effect of FTS infusions into BLA, CEA or CPU on contextual and auditory fear conditioning}

The subjects were 33 adult male rats. Rats received bilateral guide cannula implants aimed at BLA, CEA or CPU. On the day before training, rats underwent mock infusions and pre-exposure to the conditioning chambers as described for Experiment 1. On the following day, rats received bilateral infusions of either $30 \mu \mathrm{g}$ FTS per side (BLA, $n=8$; CEA, $n=8$; CPU, $n=8$ ) or vehicle ( $n=9$, split among the three cannula placements) and were returned to their home cages. Fifteen minutes later, the rats were brought to the conditioning chambers, where, after a 2-min pretrial period, they received five tone $(30 \mathrm{~s}, 85 \mathrm{~dB}$ tone, $2 \mathrm{kHz})-$ shock ( $1 \mathrm{~mA}, 2 \mathrm{~s})$ pairings (1 min ITI). On the following day, fear conditioning to the context was assessed by returning the rats to the conditioning chambers and measuring freezing during an 8-min retention test. Fear conditioning to the tone was assessed by placing the rats in a novel context (Context B) $24 \mathrm{~h}$ after the context retention test, and assessing freezing during a 6-min tone $(85 \mathrm{~dB}$, $2 \mathrm{kHz}$ ), which was delivered $2 \mathrm{~min}$ after the rats were placed in the chambers.

\section{Experiment 5: effect of intra-amygdala FTS on post-training MAPK activation in the BLA}

The subjects were 29 adult male rats (one rat was excluded from the analysis because its level of immunoreactivity was over 2.5 standard errors above the mean of its group). Rats received bilateral guide cannula implants aimed at either BLA or CEA. On the day before training, rats underwent mock infusions and pre-exposure to the conditioning chambers as described for Experiment 1. On the following day, rats received bilateral infusions of either $30 \mu \mathrm{g}$ FTS per side $(n=19)$ or vehicle $(n=10)$ and were returned to their home cages. Fifteen minutes later, the rats were put in the conditioning chambers. Three minutes after placement into the chambers, rats received 10 unsignaled footshocks $(2 \mathrm{~s}, 1 \mathrm{~mA}, 1 \mathrm{~min}$ ITI) and were then returned to their home cages. One hour later, the rats were deeply anesthetized with sodium pentobarbital $(65 \mathrm{mg} / \mathrm{kg}$, i.p.) and decapitated. The brain was removed and a punch of the BLA was taken with a 1-mm punch tool (Fine Science Tools, Foster City, CA, USA) and frozen at $-80{ }^{\circ} \mathrm{C}$ until further processing.

BLA samples were homogenized in ice-cold buffer $(150 \mathrm{mM} \mathrm{NaCl}$, $10 \mathrm{~mm}$ HEPES, pH 7.4, $10 \mathrm{~mm}$ EDTA, $10 \mathrm{~mm} \mathrm{NaF,} 1 \mu \mathrm{M}$ microcystin LR, $1 \mathrm{mM} \mathrm{Na}$ orthovanadate, $0.1 \mathrm{mM}$ PMSF, $2 \mu \mathrm{g} / \mathrm{mL}$ CLAP, a protease inhibitor cocktail containing chemostatin, levpeptin, antipain and pepstain) and centrifuged for $1 \mathrm{~min}$ at $15000 \mathrm{~g}$ to remove debris. Protein concentration in each sample was determined using a standard protein quantification procedure (Protein Assay Dye Reagent Concentrate; Bio-Rad, Hercules, CA, USA). Loading buffer was added to the supernatant and the mixture was heated at $95{ }^{\circ} \mathrm{C}$ for $5 \mathrm{~min}$. After loading equal amounts of total protein from each sample, samples were electrophoresed on $10 \%$ SDS-polyacrylamide gels and then transferred to PVDF membrane (Millipore, Bedford, MA, USA). Blots were blocked in buffer ( $1 \times$ TBS, $0.1 \%$ Tween- $20,5 \% \mathrm{w} / \mathrm{v}$ nonfat dry milk) then incubated with either anti-p44/42 MAPK or antiphospho-p44/42 MAPK (Cell Signaling Technology, Beverly, MA, USA). Blots were then incubated with either anti-rabbit or anti-mouse antibody (Pierce, Rockford, IL) and developed using enhanced chemiluminescence (SuperSignal Extended Duration Western Blotting Kit, Pierce, Rockford, IL, USA). The blots were scanned and band intensity was quantified using Adobe Photoshop. A phosphoMAPK/total MAPK ratio was calculated for each sample. This ratio was normalized to $100 \%$ in controls. Finally, phospho-MAPK/total MAPK ratios in the FTS-treated group were expressed as a percentage of those in the control group.

\section{Histology}

Histological verification of cannula placement was performed after behavioral testing in Experiments 1-3. After being overdosed with sodium pentobarbital, rats were perfused across the heart with $0.9 \%$ saline followed by $10 \%$ formalin. Brains were removed from the skull and placed in 10\% formalin for 2 days and $10 \%$ formalin/ $30 \%$ sucrose until sectioning. Coronal sections (40 $\mu \mathrm{m}$ thick, taken every $120 \mu \mathrm{m})$ were cut on a cryostat $\left(18{ }^{\circ} \mathrm{C}\right)$ and wet mounted on glass microscope slides with $95 \%$ ethanol. After drying, sections were stained with $0.25 \%$ thionin to allow visualization of cell bodies. Cannula placements were reconstructed on stereotaxic atlas templates (Swanson, 1999). Rats were excluded from statistical analyses if the cannulas were not both located in a single target structure (BLA, CEA or CPU). In all sections the tissue appeared normal and undamaged by drug infusion.

\section{Data analysis}

Freezing was calculated as a percentage of total observations, a probability estimate amenable to analysis with parametric statistics. Probability estimates of freezing were assessed through analysis of variance (ANOvA). Post-hoc comparisons in the form of Fisher's protected least significant difference tests were performed on the freezing averages after a significant omnibus $F$ ratio. All data shown in the figures are represented as means plus or minus standard errors of the means. 


\section{Results}

Intra-amygdala infusion of FTS impairs acquisition of long-term conditional fear memory in a dose-dependent manner

A photomicrograph of a representative cannula placement in the BLA is shown in Fig. 1. The tissue adjacent to the infusion site appears normal. Infusion sites for this experiment are shown in Fig. 2. Cannula placements all successfully targeted the BLA and were bilaterally symmetrical. Freezing behavior was assessed during the conditioning session to index short-term memory and during a retention test $24 \mathrm{~h}$ after conditioning to assess long-term fear memory. As shown in Fig. 3, intra-BLA infusions of FTS prior to contextual fear conditioning did not affect immediate post-shock freezing during the training session (Fig. 3a) but significantly attenuated conditional freezing in a dose-dependent manner during a retention test $24 \mathrm{~h}$ later (Fig. 3b and c). This observation was confirmed by the absence of a main effect in the ANOVA for the training session $\left(F_{3,28}=0.08\right)$ and by a significant main effect of dose $\left(F_{3,28}=3.3, P<0.05\right)$ in the ANOVA for the retention test. Post-hoc comparisons $(P<0.05)$ revealed that rats infused with $30.0 \mu \mathrm{g}$ FTS exhibited significantly less freezing during the retention test than rats receiving either $3.0 \mu \mathrm{g}$ or vehicle. These results indicate that FTS does not impair immediate post-shock freezing during conditioning but impairs consolidation of a long-term conditional fear memory assessed $24 \mathrm{~h}$ after training.

\section{Intra-amygdala infusions of FTS do not impair expression of long-term fear memory}

Although rats administered FTS exhibited normal immediate postshock freezing on the conditioning day, we examined whether FTS affects the expression of conditional freezing during retention testing. To determine whether intra-amygdala FTS infusions impair the expression of long-term conditional fear memory, rats from the previous experiment were given new group assignments (counterbalancing for previous group assignment) and were retrained drug-free. On the following day, rats received an infusion of either FTS or vehicle into the BLA $15 \mathrm{~min}$ prior to a retention test. As shown in Fig. 4, FTS had no effect on expression of long-term conditional fear memory. This was confirmed by the ANOVA with a non-significant group effect $\left(F_{1,17}=0.003\right)$. This result suggests that Ras function is not required for expression of either short- or long-term fear memory.

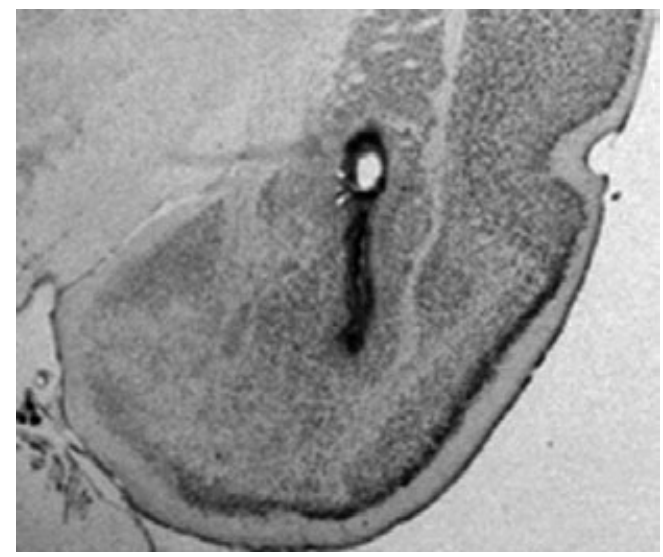

FIG. 1. Cannula placements in the basolateral amygdala. Photomicrograph showing a representative cannula placement in the basolateral amygdala (BLA). Other than the damage caused by the guide cannula and injector, tissue in the region of the cannula was normal after FTS infusion.

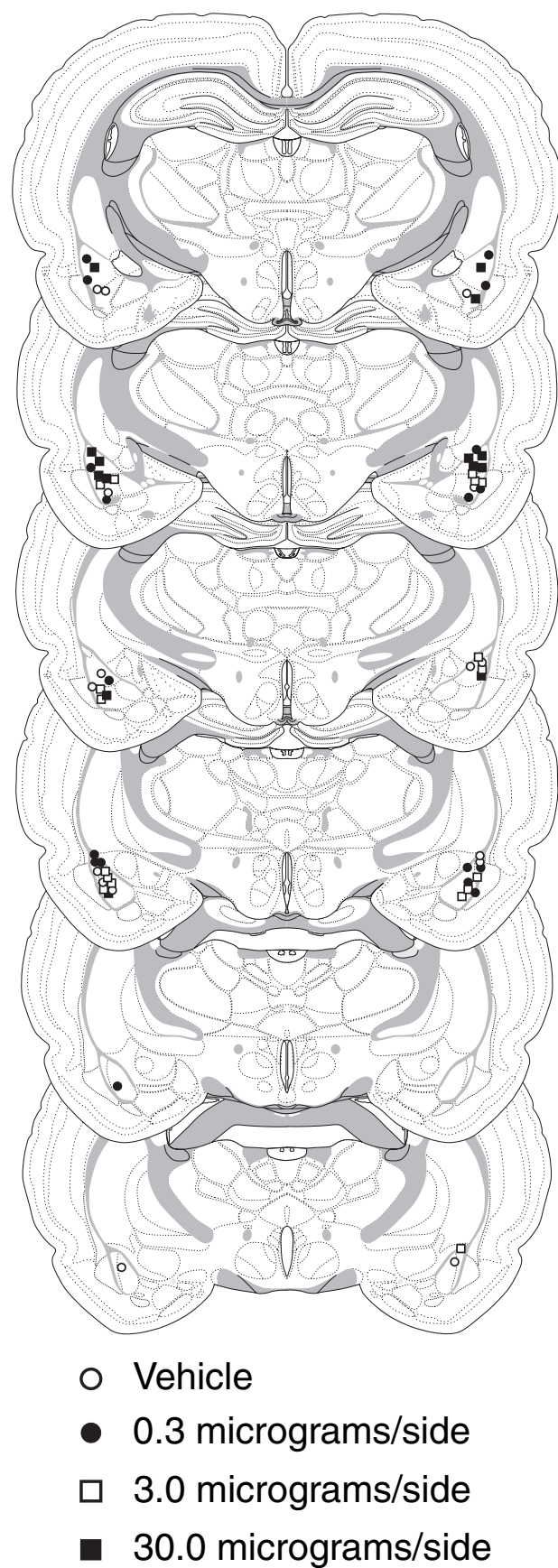

FIG. 2. Schematic diagram illustrating infusion sites for Experiment 1. Coronal sections are adapted from Swanson (1999).

Furthermore, normal conditioning and fear expression in rats that had previously received drug infusions suggests that our infusion procedure, including volume and rate, does not damage the BLA.

\section{Intra-amygdala infusions of FTS do not block acquisition of short-term conditional fear memory}

Although FTS had no effect on post-shock freezing during the conditioning session, we recognize that post-shock freezing is not the most accurate measure of short-term memory for fear conditioning. To assess short-term memory and long-term memory in the same animals, we used auditory fear conditioning and exposed the animal to the tone 


\section{a}

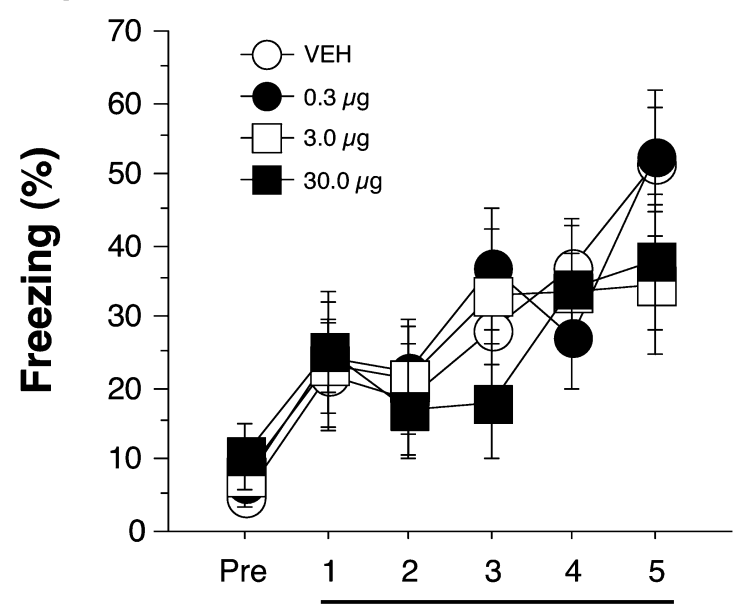

b

Trial

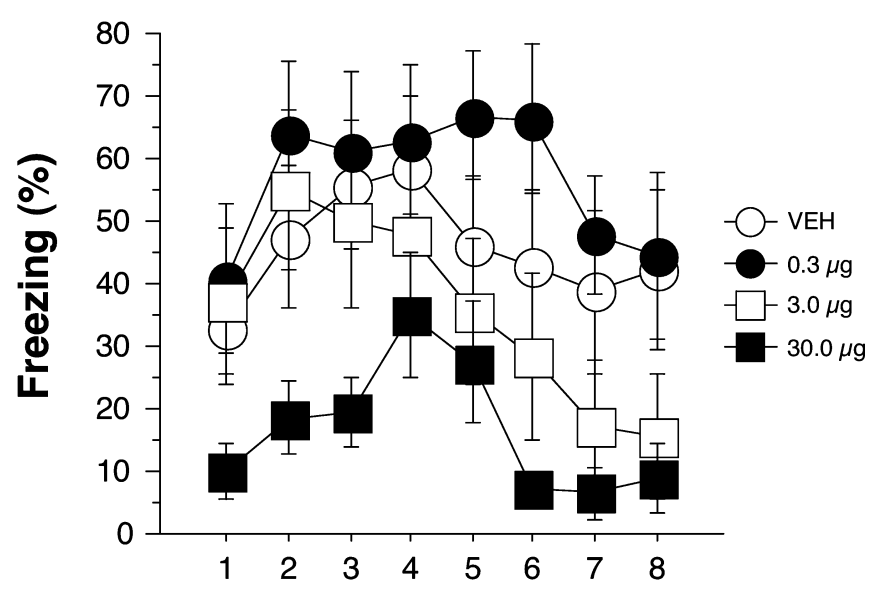

C

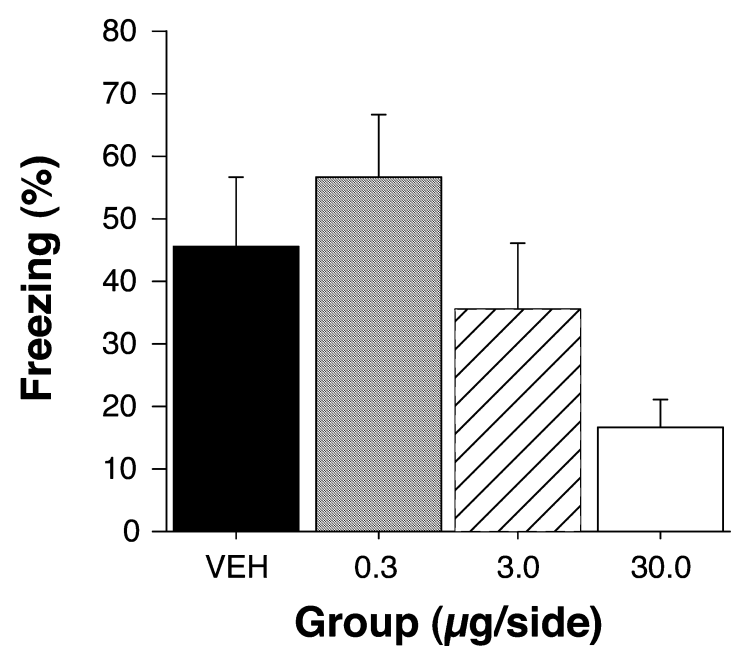

FIG. 3. Dose-response analysis of the effect of intra-BLA FTS infusion on contextual fear conditioning. Mean percentage of freezing ( \pm SEM) exhibited on the training day (a), both prior to shock (Pre) and during the 1-min interval following each footshock (Trials 1-5) and during an 8-min context retention test on the following day (b and c) in rats infused with varying doses of FTS 15 min prior to training.

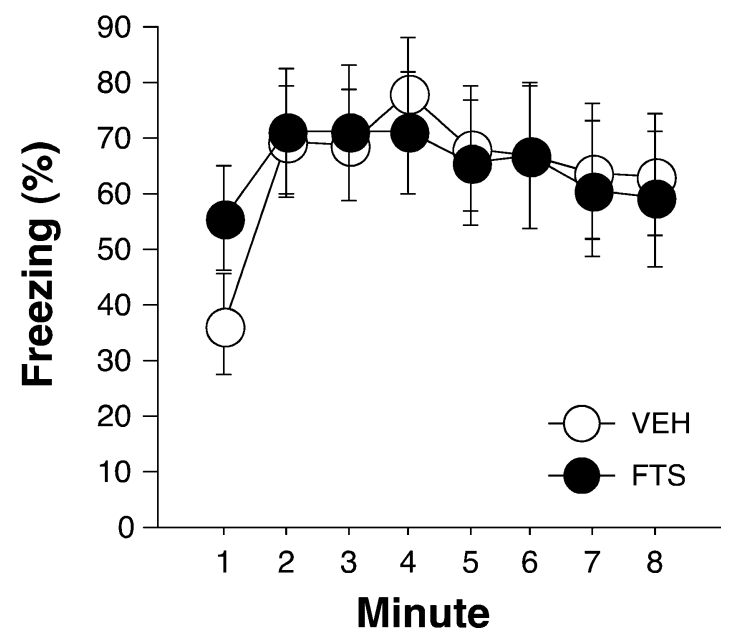

FIG. 4. Analysis of the effect of intra-BLA FTS infusion on expression of long-term memory for contextual fear conditioning. Mean percentage of freezing $( \pm$ SEM) exhibited during an 8-min retention test in rats infused with either vehicle or $30 \mu \mathrm{g}$ FTS per side $15 \mathrm{~min}$ prior to the retention test.

conditioning stimulus either 1 or $24 \mathrm{~h}$ after conditioning. This procedure has been used to establish a selective role for several molecular cascades in the amygdala in long-term memory, as opposed to short-term memory (e.g. Schafe et al., 2000a). Cannula placements all successfully targeted the BLA and were bilaterally symmetrical (data not shown). As shown in Fig. 5, a preconditioning FTS infusion into the BLA had no effect on conditional freezing during a short-term memory test $1 \mathrm{~h}$ after conditioning but significantly attenuated conditional freezing during a long-term memory test $24 \mathrm{~h}$ after conditioning $(P<0.05)$. These results indicate that intra-BLA FTS infusion has no effect on acquisition of short-term fear memory but significantly impairs consolidation of long-term memory.

\section{Post-training infusions of FTS have no effect on consolidation of long-term conditional fear memory}

To determine the time window for Ras activation during contextual fear conditioning, rats received infusions of FTS or vehicle into the BLA at one of three post-training time points: immediately, $1 \mathrm{~h}$ or $6 \mathrm{~h}$

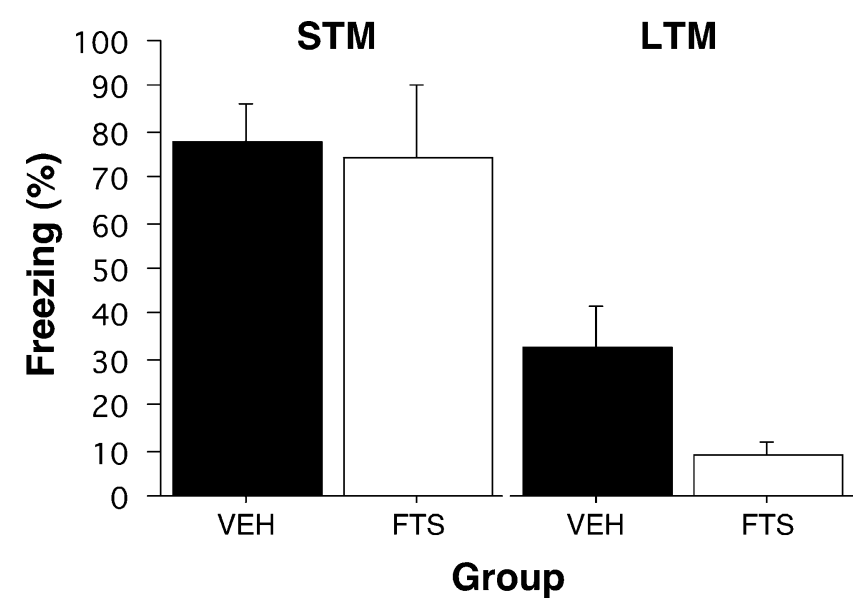

FIG. 5. Effect of intra-BLA FTS infusion on acquisition of short-term and long-term auditory fear memory. Mean percentage of freezing ( \pm SEM) exhibited during a short-term memory (STM) test $1 \mathrm{~h}$ after conditioning and long-term memory (LTM) test $24 \mathrm{~h}$ after conditioning in rats infused with either vehicle or $30 \mu \mathrm{g}$ FTS per side 15 min prior to fear conditioning. 

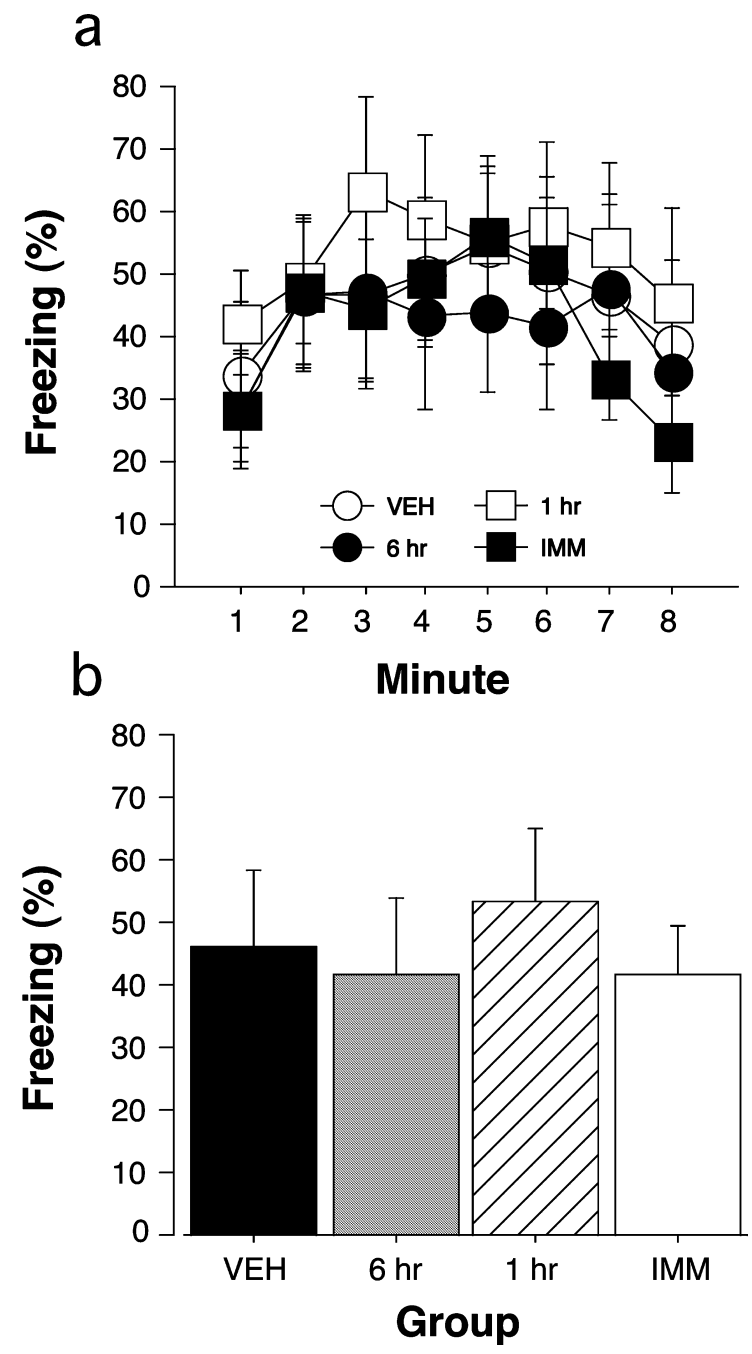

FIG. 6. Effect of intra-BLA FTS infusions immediately, $1 \mathrm{~h}$ or $6 \mathrm{~h}$ after training on acquisition of long-term memory for contextual fear conditioning. ( $a$ and $b)$ Mean percentage of freezing ( \pm SEM) exhibited during an 8-min retention test in rats infused with either vehicle or $30 \mu \mathrm{g}$ FTS per side immediately, $1 \mathrm{~h}$ or $6 \mathrm{~h}$ after training. There were no differences observed between rats infused with vehicle at each of the three time points, so the three groups were collapsed into a single 'vehicle' group.

after training. Cannula placements all successfully targeted the BLA and were bilaterally symmetrical (data not shown). As shown in Fig. 6, there were no significant differences in conditional freezing between any of the groups during a retention test $24 \mathrm{~h}$ after conditioning. This was indicated in the ANOVA by a non-significant group effect $\left(F_{2,21}=0.69\right)$. Thus, post-training FTS administration had no effect on consolidation of long-term conditional fear memory, suggesting a short time window for Ras activation during the acquisition of fear conditioning.

\section{Effect of FTS on fear conditioning is specific for the BLA and FTS disrupts both auditory and contextual fear conditioning}

To determine the possible anatomical and modality specificity of the inhibitory effect of FTS on fear conditioning, rats received infusions of vehicle or FTS into the BLA, CEA or CPU prior to fear conditioning and the effect of FTS on both contextual and auditory fear conditioning was assessed. Infusion sites for this experiment are shown in Fig. 7. All cannula placements were accurate and bilaterally symmetrical. As

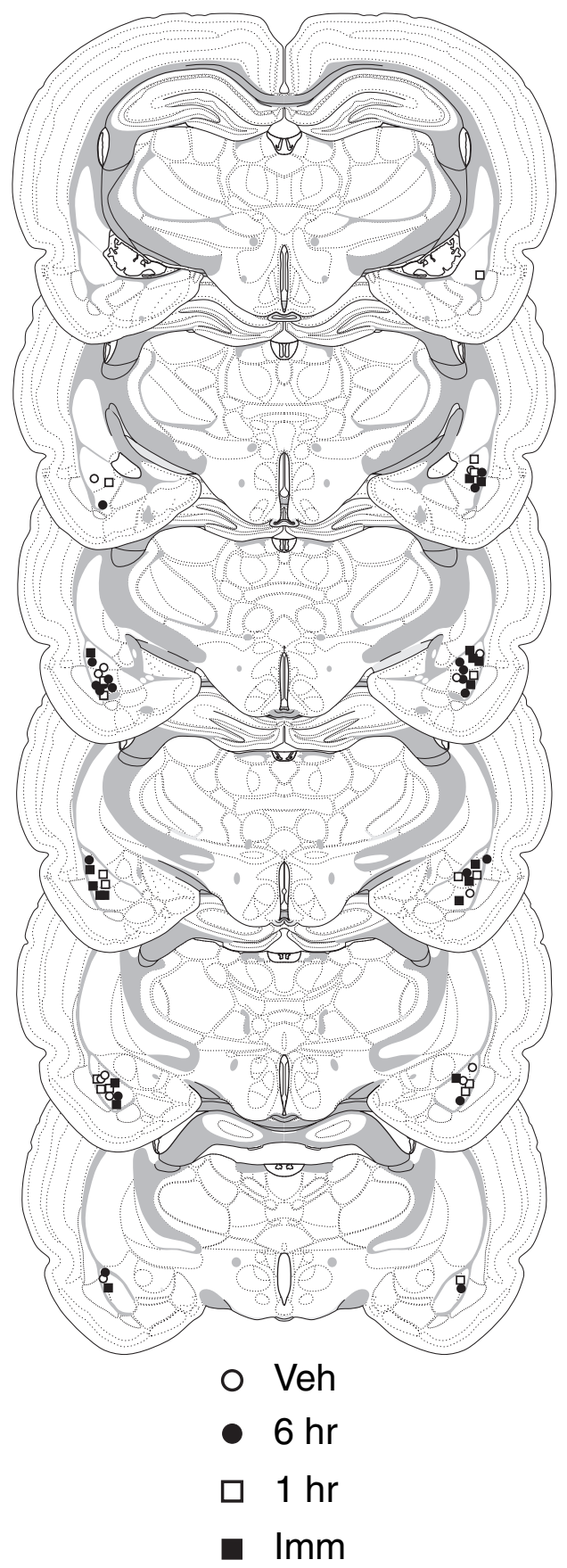

FIG. 7. Schematic diagram illustrating infusion sites for Experiment 4. Coronal sections adapted from Swanson (1999). BLA, basolateral amygdala; $\mathrm{CEA}$, central nucleus of the amygdala; CPU, caudate-putamen.

before, FTS did not affect immediate post-shock freezing on the conditioning day (data not shown). As shown in Fig. 8, rats receiving intra-BLA infusions of FTS exhibited significantly less freezing than all other groups during both context (Fig. $8 \mathrm{a}$ and $\mathrm{b}$ ) and tone retention tests (Fig. $8 \mathrm{c}$ and d). These observations were confirmed by the ANOVA for the context test $\left(F_{3,29}=8.68, P<0.0005\right)$ and for the tone test $\left(F_{3,29}=3.03, P<0.05\right)$ and by post-hoc comparisons $(P<0.05)$. In the first minute of the tone, rats receiving intra-CEA FTS infusions exhibited less freezing than vehicle controls and rats receiving intraCPU FTS. These differences, however, were not significant, and over the course of the 6-min tone rats receiving intra-CEA FTS still 
a

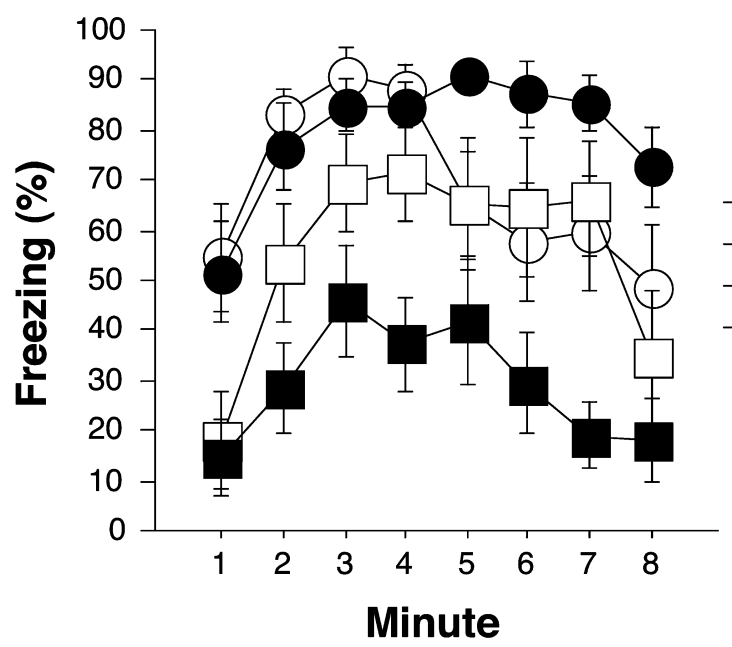

C

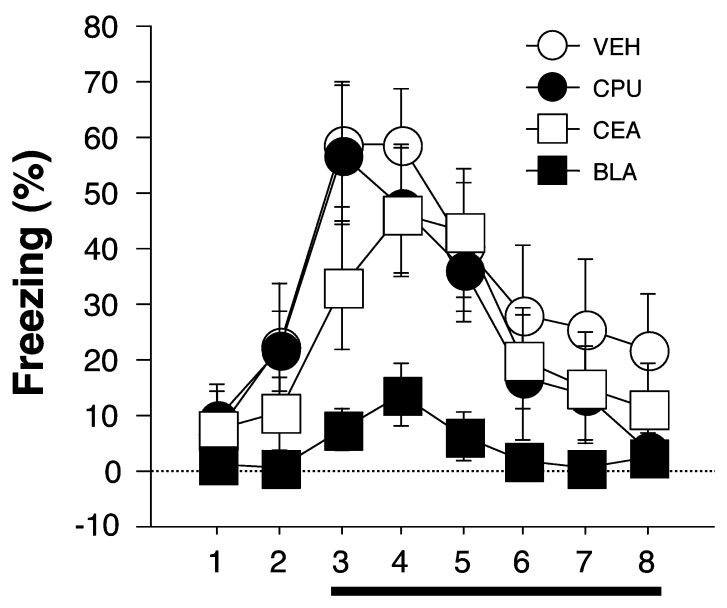

Minute b

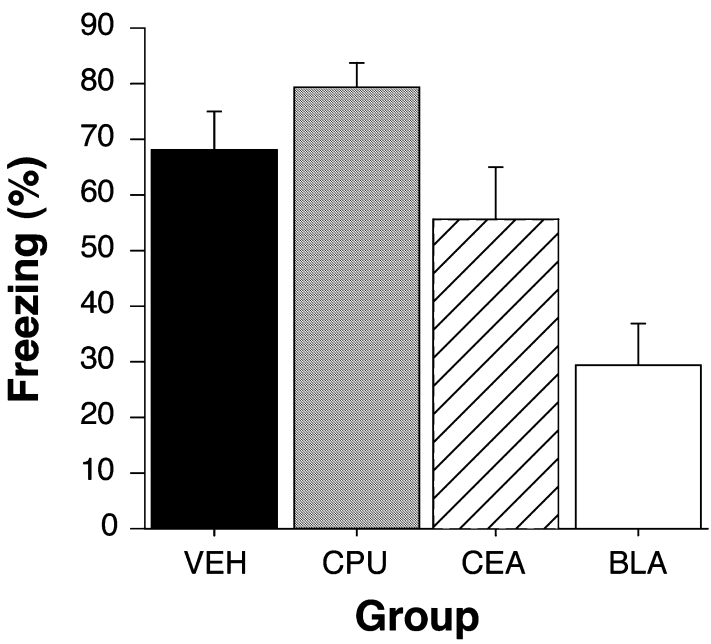

d

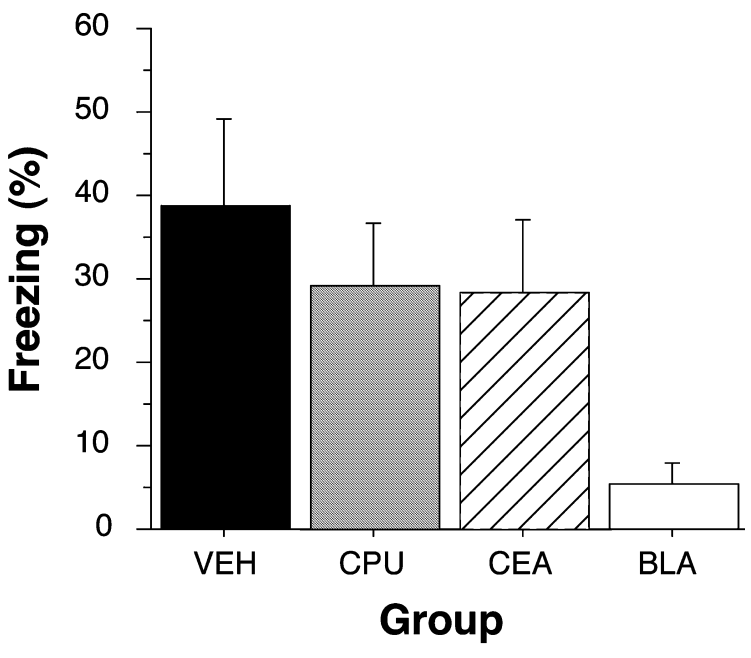

FIG. 8. Effects of FTS infusions into BLA, CEA or CPU on contextual and auditory fear conditioning. Mean percentage of freezing ( \pm SEM) exhibited during an 8-min context retention test in the training context (Context $\mathrm{A}$ ) (a and b), and the 8-min tone retention test in a novel context $($ Context $\mathrm{B})(\mathrm{c}$ and $\mathrm{d}$ ) in rats receiving an infusion of either vehicle or $30 \mu \mathrm{g}$ FTS per side into the basolateral amygdala (BLA) central nucleus of the amygdala (CEA) or caudate-putamen (CPU) 15 min prior to training. There were no differences observed between rats infused with vehicle into the BLA, CEA or CPU for either contextual or tone freezing, and thus the three groups were collapsed into a single 'vehicle' group for both measures. The presence of the tone is indicated by the black bar below the $x$-axis.

performed significantly better than those receiving intra-BLA FTS $(P<0.05)$. In the first 2 min of the context test, rats receiving intraCEA FTS exhibited significantly lower freezing than vehicle controls and intra-CPU FTS controls $(P<0.05)$. However, as in the tone test, over the course of the entire test these rats still performed significantly better than those receiving intra-BLA FTS $(P<0.05)$ and did not differ significantly from controls. The potential drug effect in CEA in these experiments may be the result of diffusion of FTS into the BLA (given the proximity of the two structures) or may reflect a minor effect of FTS in the CEA through Ras inhibition. Overall, the results from this experiment indicate that the effect of FTS on fear conditioning is specific for the BLA, and that both auditory and contextual fear memory are disrupted by FTS infusion.

\section{FTS reduces phospho-MAPK levels in the BLA}

Fear conditioning increases MAPK phosphorylation in the lateral nucleus of the amygdala (LA), which is contained in the BLA (Schafe et al., 2000a). To determine whether FTS inhibits MAPK activation in the BLA, we used Western blotting to measure post-training levels of phospho-MAPK in the BLA. Rats received intra-amygdala infusions of either vehicle or FTS prior to training, which consisted of ten unsignaled footshocks ( $1 \mathrm{~mA}, 2 \mathrm{~s}, 1 \mathrm{~min}$ ITI). The rats were killed $1 \mathrm{~h}$ after training and a punch of the BLA was taken and subjected to Western blot analysis. The size of the tissue punch necessitated measuring phospho-MAPK for the entire BLA. As shown in Fig. 9, FTS significantly reduced levels of phospho-MAPK in the BLA. This was confirmed by the ANOVA with a significant main effect of group $\left(F_{1,28}=5.78, P<0.05\right)$. No-shock controls were not used in the experiment, although others have shown that fear conditioning increases MAPK phosphorylation in the BLA (Schafe et al., 2000a).

\section{Discussion}

In the present study, we examined the role of the small GTPase Ras in acquisition and expression of long-term conditional fear memory in rats 


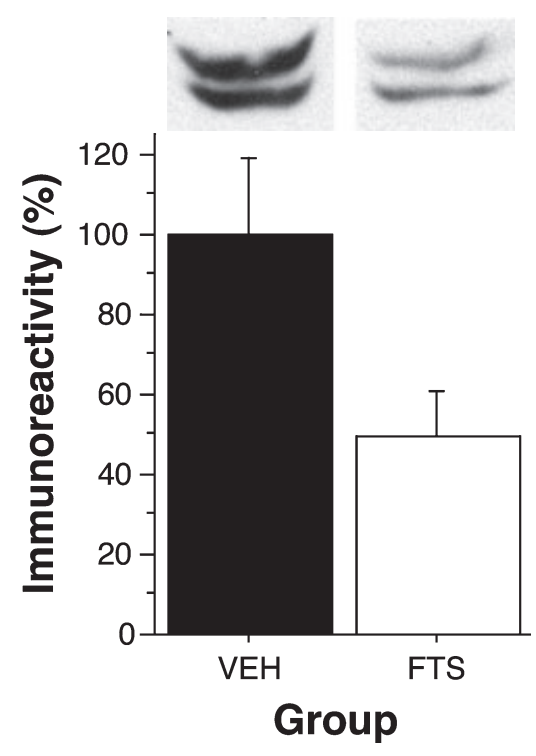

FIG. 9. Analysis of the effect of intra-amygdala FTS on MAPK activation in the BLA after fear conditioning. Representative blots and mean percentage ( \pm SEM) phospho-MAPK immunoreactivity from BLA punches taken $1 \mathrm{~h}$ after training from rats given intra-amygdala infusions of either vehicle or $30 \mu \mathrm{g}$ FTS per side $15 \mathrm{~min}$ prior to the training session. Immunoreactivity in FTStreated rats is expressed as a percentage of controls.

by using intra-amygdala infusions of the selective Ras antagonist FTS. The results indicate that infusion of FTS into the BLA prior to fear conditioning impairs acquisition but not expression of long-term memory for contextual fear conditioning in a dose-dependent manner. Intra-BLA infusions also disrupted acquisition of long-term memory for auditory fear conditioning, and post-training infusions of FTS into the BLA were ineffective at impairing consolidation of fear memory. The effect of FTS on fear conditioning was specific for the BLA in so far as intra-CEA infusions were ineffective at impairing consolidation of fear memory for auditory fear conditioning. Importantly, FTS affected neither immediate post-shock freezing during the conditioning session nor expression of long-term fear memory when infused prior to a retention test. Finally, intra-amygdala infusions of FTS significantly reduced post-training levels of phospho-MAPK in the BLA.

Our results provide several new insights into the role of Ras in amygdaloid synaptic plasticity and fear memory. Although several studies have provided indirect evidence for an involvement of Ras in amygdaloid synaptic plasticity and long-term fear memory (Brambilla et al., 1997; Schafe et al., 2000a; Dhaka et al., 2003), ours is the first to provide direct evidence for its involvement in fear memory acquisition using a selective Ras antagonist and demonstrating its inhibitory effect on acquisition of fear memory. Our experiments reveal that Ras activity in the BLA is required for consolidation but not expression of long-term conditional fear memory. Importantly, intra-BLA FTS infusion prior to conditioning had no effect on postshock freezing during the conditioning session nor freezing during a short-term auditory fear memory test $1 \mathrm{~h}$ after training. Thus, FTS has no effect on expression of short-term fear memory.

Although pretraining FTS infusions attenuated fear memory, posttraining infusions had no effect on the consolidation of these memories. The ineffectiveness of post-training infusions at blocking consolidation of fear memory suggests a short period of activation for Ras during fear conditioning. The inability of even an immediate post-training infusion suggests that fear conditioning rapidly generates NMDA receptor activation and subsequent Ras activation, and that Ras activation is essential during but not shortly after fear conditioning. By contrast, some drugs are effective at blocking consolidation of fear memory when infused into the BLA after conditioning. Infusion of a protein synthesis inhibitor (Maren et al., 2003) or a protein kinase A inhibitor (Schafe \& Ledoux, 2000) immediately after fear conditioning blocks consolidation of memory for fear conditioning. It is likely that these cellular events occur further downstream from Ras and take place over a longer period of time, making them susceptible to post-conditioning pharmacological inhibition. Additional research is needed to determine more precisely the sequence and timing of Ras/MAPK activation in the BLA as a result of fear conditioning.

Although previous studies have implicated Ras in fear memory, they failed to determine the anatomical localization of Ras/MAPK activity critical for fear conditioning. For example, studies employing pharmacological inhibition of MAPK activation have not targeted the BLA and CEA independently (Schafe et al., 2000). Our results suggest that for acquisition of long-term fear memory Ras activity is required in the BLA but not in the CEA. Additional studies are needed to confirm our finding that Ras/MAPK activity in the CEA is not required for consolidation of fear memory. Our results are consistent with an earlier study from our laboratory which provided evidence suggesting that the BLA, not the CEA, is the critical locus for intracellular mechanisms underlying acquisition of fear memory (Goosens et al., 2000). H7, an inhibitor of both protein kinase C and cAMP-dependent protein kinase A, impaired acquisition of longterm conditional fear memory when infused prior to conditioning into the BLA but not when infused into the CEA.

Immunohistochemical studies have determined that fear conditioning induces a significant increase in phospho-MAPK in the LA which peaks $1 \mathrm{~h}$ after conditioning (Schafe et al., 2000). We demonstrated that by inhibiting Ras activation with FTS, MAPK activation in the BLA after fear conditioning was reduced. It seems reasonable to assume that the inhibitory effect of FTS on acquisition of conditional fear memory is due, at least in part, to its downstream inhibition of MAPK activation. Thus, our data provide additional evidence to support the notion that the Ras/MAPK pathway is essential for amygdaloid synaptic plasticity and fear memory, while providing new insights into the role of Ras in these processes.

An important question concerns the specificity of FTS for Ras. FTS disrupts the membrane anchorage of Ras and dislodges it from the cell membrane, thereby facilitating its increased degradation (Haklai et al., 1998). FTS has been shown to inhibit MAPK activation in cell cultures (Gana-Weisz et al., 1997) and in the brain (Dhaka et al., 2003). The literature on FTS suggests that it is highly selective for Ras (Kloog \& Cox, 2004). FTS inhibits the growth of cultured ErbB2- or Ras-transformed cells, growth of which is dependent on normal Ras signaling, but not v-Raf-transformed cells, growth of which is not dependent on Ras (Haklai et al., 1998). The same study demonstrated that FTS has no effect on cells expressing a form of Ras that lacks the normal post-translational modification of farnesylation. The study also determined that FTS does not affect another class of G-proteins - the G $\beta$ subunit. Jansen et al. (1999) demonstrated that in human melanoma cells FTS causes a decrease in amounts of Ras, but not related proteins Rac or RhoA. Despite the high degree of specificity determined by cell culture studies, it is difficult to be certain that FTS has a similar degree of specificity in brain tissue. Although our study demonstrates that intra-amygdala infusions of FTS are capable of causing downstream inhibition of the Ras/MAPK pathway, it is unclear what nonspecific effects FTS may have, if any, at the concentrations used in our experiment. Additional experiments are necessary to determine whether FTS has an effect on any other protein signaling in the brain. 
The effectiveness of FTS in disrupting fear memory raises interesting questions. What effect would intra-amygdala FTS have on induction of amygdaloid LTP both in vivo and in vitro? Furthermore, the results of our study raise additional questions regarding Ras activation and signaling in the amygdala. What upstream signals regulate Ras activation and signaling? Mice deficient in RasGRF, which interacts with and activates Ras, show significant deficits in fear conditioning and amygdaloid LTP (Brambilla et al., 1997). RasGRF can be activated by $\mathrm{Ca}^{2+}$ influx (Farnsworth et al., 1995) and recently it was demonstrated that RasGRF binds directly to the NR2B subunit of NMDA receptors (Krapivinsky et al., 2003). This interaction provides a direct link between NMDA receptor activation and resultant $\mathrm{Ca}^{2+}$ influx and activation of the Ras/MAPK signaling pathway. Further research is necessary to understand better the regulation and activation of Ras/MAPK signaling in amygdaloid synaptic plasticity and fear memory. In summary, our study provides further evidence for the involvement of Ras in amygdaloid synaptic plasticity and acquisition of fear memory and highlights the importance of Ras/MAPK signaling in the amygdala.

\section{Acknowledgements}

This work was supported by a grant to S.M. (NIMH R01MH065961) and a predoctoral fellowship from the American Psychological Association Diversity Program in Neuroscience awarded to S.M.M. We would like to thank Jose Esteban for assistance with Western blotting procedures.

\section{Abbreviations}

BLA, basolateral amygdala; CEA, central nucleus of the amygdala; CPU, caudate putamen; FTS, farnesylthiosalicylic acid; ITI, intertrial interval; LA, lateral nucleus of the amygdala; LTP, long-term potentiation; MAPK, mitogenactivated protein kinase.

\section{References}

Adams, J.P. \& Sweatt, J.D. (2002) Molecular psychology: roles for the ERK MAP kinase cascade in memory. Annu. Rev. Pharmacol. Toxicol., 42, 142-163.

Bauer, E.P., Schafe, G.E. \& LeDoux, J.E. (2002) NMDA receptors and L-type voltage-gated calcium channels contribute to long-term potentiation and different components of fear memory formation in the lateral amygdala. J. Neurosci., 22, 5239-5249.

Blair, H.T., Schafe, G.E., Bauer, E.P., Rodrigues, S.M. \& LeDoux, J.E. (2001) Synaptic plasticity in the lateral amygdala: a cellular hypothesis of fear conditioning. Learn. Mem., 8, 229-242.

Blum, S., Moore, A.N., Adams, F. \& Dash, P.K. (1999) A mitogen-activated protein kinase cascade in the CA1/CA2 subfield of the dorsal hippocampus is essential for long-term spatial memory. J. Neurosci., 19, 3535-3544.

Brambilla, R., Gnesutta, N., Minichiello, L., White, G., Roylance, A.J., Herron, C.E., Ramsey, M., Wolfer, V.C., Cestari, V., Rossi-Arnaud, C., Grant, S.E., Chapman, P.F., Lipp, H.-P., Sturani, E. \& Klein, R. (1997) A role for the Ras signaling pathway in synaptic transmission and long-term memory. Nature, 390, 281-286.

Clugnet, M.C. \& LeDoux, J.E. (1990) Synaptic plasticity in fear conditioning circuits: induction of LTP in the lateral nucleus of the amygdala by stimulation of the medial geniculate body. J. Neurosci., 10, 2918-2924.

Dhaka, A., Costa, R.M., Hu, H., Irwin, D.K., Patel, A., Kornblum, H.I., Silva, A.J., O’Dell, T.J. \& Colicelli, J. (2003) The RAS effector RIN1 modulates the formation of aversive memories. J. Neurosci., 23, 748-757.

English, J.D. \& Sweatt, J.D. (1996) Activation of p42 mitogen-activated protein kinase in hippocampal long-term potentiation. J. Biol. Chem., 271, 24329-24332.

English, J.D. \& Sweatt, J.D. (1997) A requirement for the mitogen-activated protein kinase cascade in hippocampal long-term potentiation. J. Biol. Chem., 272, 19103-19106.

Fanselow, M. \& LeDoux, J.E. (1999) Why we think plasticity underlying Pavlovian fear conditioning occurs in the basolateral amygdala. Neuron, 23, 229-232.
Farnsworth, C.L., Freshney, N.W., Rosen, L.B., Ghosh, A., Greenberg, M.E. \& Feig, L.A. (1995) Calcium activation of Ras mediated by neuronal exchange factor Ras-GRF. Nature, 376, 524-527.

Gana-Weisz, M., Haklai, R., Marciano, D., Egozi, Y., BenBaruch, G. \& Kloog, Y. (1997) The Ras antagonist S-farnesylthiosalicylic acid induces inhibition of MAPK activation. Biochem. Biophys. Res. Commun., 239, 900-904.

Goosens, K.A., Holt, W. \& Maren, S. (2000) A role for amygdaloid PKA and $\mathrm{PKC}$ in the acquisition of long-term conditional fear memories in rats. Behav. Brain Res., 114, 145-152.

Haklai, R., Gana-Weisz, G., Elad, G., Paz, A., Marciano, D., Egozi, Y., BenBaruch, G. \& Kloog, Y. (1998) Dislodgment and accelerated degradation of Ras. Biochemistry, 37, 1306-1314.

Huang, Y.Y. \& Kandel, E.R. (1998) Postsynaptic induction and PKAdependent expression of LTP in the lateral amygdala. Neuron, 21, 69-78.

Impey, S., Obrietan, K. \& Storm, D.R. (1999) Making new connections: role of ERK/MAP kinase signaling in neuronal plasticity. Neuron, 23, 11-14.

Jansen, B., Schlagbauer-Wadl, H., Kahr, H., Ress, E., Mayer, B., Eichler, H.G., Ben-David, E., Kloog, Y. \& Wolff, K. (1999) Novel Ras antagonist blocks human melanoma growth. Proc. Natl Acad. Sci. USA, 96, 14019-14024.

Kloog, Y. \& Cox, A.D. (2004) Prenyl-binding domains: potential targets for Ras inhibitors and anti-cancer drugs. Semin. Cancer Biol., 14, 253-261.

Krapivinsky, G., Krapivinsky, L., Manasian, Y., Ivanov, A., Tyzio, R., Pellegrino, C., Ben-Ari, Y., Clapham, D.E. \& Medina, I. (2003) The NMDA receptor is coupled to the ERK pathway by a direct interaction between NR2B and RasGRF1. Neuron, 40, 775-784.

Lee, H. \& Kim, J.J. (1998) Amygdalar NMDA receptors are critical for new fear learning in previously fear-conditioned rats. J. Neurosci., 18, 8444-8454.

Lee, Y., Walker, D. \& Davis, M. (1996) Lack of a temporal gradient of retrograde amnesia following NMDA-induced lesions of the basolateral amygdala assessed with the fear-potentiated startle paradigm. Behav. Neurosci., 10, 836-839.

Maren, S. (1999) Long-term potentiation in the amygdala: a mechanism for emotional learning and memory. Trends Neurosci., 2, 561-567.

Maren, S. (2001) Neurobiology of Pavlovian fear conditioning. Annu. Rev. Neurosci., 24, 897-931.

Maren, S., Aharonov, G., Stote, D.L. \& Fanselow, M.S. (1996) N-methyl-Daspartate receptors in the basolateral amygdala are required for both acquisition and expression of conditional fear in rats. Behav. Neurosci., 110, $1365-1374$.

Maren, S. \& Fanselow, M.S. (1995) Synaptic plasticity in the basolateral amygdala induced by hippocampal formation stimulation in vivo. J. Neurosci., 15, 7548-7564.

Maren, S., Ferrario, C.R., Corcoran, K.A., Desmond, T.J. \& Frey, K.A. (2003) Protein synthesis in the amygdala, but not the auditory thalamus, is required for consolidation of Pavlovian fear conditioning in rats. Eur. J. Neurosci., 18, 3080-3088.

Maren, S. \& Quirk, G.J. (2004) Neuronal signaling of fear memory. Nat. Rev. Neurosci., 5, 844-852.

Mazzuccheli, C. \& Brambilla, R. (2000) Ras-related and MAPK signaling in neuronal plasticity and memory formation. Cell. Mol. Life Sci., 57, 604-611.

McKernan, M.G. \& Shinnick-Gallagher, P. (1997) Fear conditioning induces a lasting potentiation of synapses in vitro. Nature, 390, 607-611.

Miserendino, M.J., Sananes, C.B., Melia, K.R. \& Davis, M. (1990) Blocking of acquisition but not expression of conditioned fear-potentiated startle by NMDA antagonists in the amygdala. Nature, 345, 716-718.

Niv, H., Gutman, O., Henis, Y.I. \& Kloog, Y. (1999) Membrane interactions of a constitutively active GFP-Ki-Ras 4B and their role in signaling. Evidence from lateral mobility studies. J. Biol Chem., 274, 1606-1613.

Rogan, M.T. \& LeDoux, J.E. (1995) LTP is accompanied by commensurate enhancement of auditory evoked responses in a fear conditioning circuit. Neuron, 15, 127-136.

Rogan, M.T., Staubli, U.V. \& LeDoux, J.E. (1997) Fear conditioning induces associative long-term potentiation in the amygdala. Nature, 390, 604-607.

Schafe, G.E., Atkins, C.M., Swank, M.W., Bauer, E.P., Sweatt, J.E. \& Ledoux, J.E. (2000) Activation of ERK/MAPK in the amygdala is required for memory consolidation of Pavlovian fear conditioning. J. Neurosci., 20, $8177-8187$.

Schafe, G.E. \& Ledoux, J.E. (2000) Memory consolidation of auditory Pavlovian fear conditioning requires protein synthesis and protein kinase A in the amygdala. J. Neurosci., 20, RC96.

Schafe, G.E., Nader, K., Blair, H.T. \& Ledoux, J.E. (2001) Memory consolidation of Pavlovian fear conditioning: a cellular and molecular perspective. Trends Neurosci., 24, 540-546.

Swanson, L.W. (1999) Brain maps: Structure of the Rat Brain, $2^{\text {nd }}$ Ed. Elsevier, Amsterdam. 